\title{
Avaliação das emissões otoacústicas em pacientes submetidos a tratamento quimioterápico em hospital de referência em oncologia pediátrica no Pará
}

\author{
Evaluation of otoacoustic emissions in patients submitted to chemotherapeutic treatment in \\ hospital of reference in pediatric oncology of Para
}

Evaluación de emisiones otoacústicas en pacientes sometidos a tratamiento quimioterápico en hospital dereferencia enoncologiapediátrica en el Pará

Cássia Maria Oliveira de Oliveira ${ }^{1 *}$, Erika Baptista Luiz Badarane ${ }^{1},^{2}$.

\section{RESUMO}

Objetivo: Avaliar as emissões otoacústicas (EOA) em pacientes submetidos a tratamento quimioterápico em hospital de referência em oncologia pediátrica no Pará. Métodos: O estudo é longitudinal, prospectivo e de coorte, realizado no Hospital Oncológico Infantil Octávio Lobo e no Hospital Universitário Bettina Ferro de Souza, obtendo-se dados dos exames de EOA transientes, em pacientes atendidos no ambulatório de oncologia pediátrica no mês de outubro de 2018, ao início do tratamento e durante o uso de quimioterápicos. $\mathrm{Na}$ aplicação da estatística descritiva, foram construídas tabelas e gráficos e a estatística analítica foi utilizada para avaliar os resultados das variáveis categóricas, através dos Testes G e Qui-Quadrado. Resultados: Foram avaliados vinte pacientes. Houve predomínio do sexo masculino, com maior prevalência de EOA presentes. Dentre os quimioterápicos, prevaleceram as platinas e derivados. A relação entre a EOA com o quimioterápico utilizado, resultou em EOA presentes em uso de platinas e derivados. Demonstrou- se que o tempo não influencia o resultado. Conclusão: Houve prevalência da presença de EOA no início do tratamento, sem relação direita com o tempo de exposição e a dosagem da droga utilizada, sendo a cisplatina o principal quimioterápico indutor de ototoxicidade.

Palavras-chave: Cisplatino, Emissões otoacústicas, Quimioterapia, Células ciliadas.

\begin{abstract}
Objective: To evaluate the otoacoustic emissions in patients submitted to chemotherapy treatment in hospital of reference in pediatric oncology of Pará. Methods: The study was longitudinal, prospective and cohort study at the Octávio Lobo Infant Oncology Hospital and at the University Hospital Bettina Ferro de Souza, obtaining data from the transient OAEs in patients attended at the pediatric oncology clinic in October 2018, at the start of treatment and during the use of chemotherapy. In the application of descriptive statistics, tables and graphs were constructed and the analytical statistic was used to evaluate the results of the categorical variables through the $G$ and Chi-Square Tests. Results: Twenty patients were evaluated. There was a predominance of males, with a higher prevalence of OAE present. Among the chemotherapeutics, the platinas and derivatives were prevalent. The relationship between OAE and chemotherapy used resulted in OAE present in the use of platinum and derivatives. It was demonstrated that the time does not influence the result. Conclusion: There was a prevalence of OAE at the beginning of the treatment, with no relation to the exposure time and dosage of the drug used, and cisplatin the main chemotherapeutic agent inducing ototoxicity.
\end{abstract}

Keywords: Cisplatin, Otoacoustic emissions, Drug terapy, Outer hair cells.

\footnotetext{
${ }^{1}$ Hospital Universitário Bettina Ferro de Souza, Belém - Pará.

${ }^{2}$ Hospital Oncológico Infantil Octávio Lobo, Belém- Pará. * E-mail: cassia.oliveira@gmail.com
} 


\section{RESUMEN}

Objetivo: Evaluar las emisiones otoacústicas (EOA) en pacientes sometidos a tratamiento quimioterápico en un hospital de referencia en oncología pediátrica en Pará. Métodos: El estudio es longitudinal, prospectivo y de cohorte, realizado en el Hospital Oncológico Infantil Octavio Lobo y en el Hospital Universitario Bettina Ferro de Souza, los pacientes atendidos en el ambulatorio de oncología pediátrica en el mes de octubre de 2018, al inicio del tratamiento y durante el uso de quimioterápicos. En la aplicación de la estadística descriptiva, se construyeron tablas y gráficos y la estadística analítica fue utilizada para evaluar los resultados de las variables categóricas, a través de las pruebas $G$ y Qui-cuadrado. Resultados: Se evaluaron veinte pacientes. Se observó predominio del sexo masculino, con mayor prevalencia de EOA presentes. Entre los quimioterápicos, prevalecieron las platinas y derivados. La relación entre la EOA con el quimioterápico utilizado, resultó en EOA presentes en uso de platinas y derivados. Se demostró que el tiempo no influye en el resultado. Conclusión: Hubo prevalencia de la presencia de EOA al inicio del tratamiento, sin relación derecha con el tiempo de exposición y la dosificación del fármaco utilizado, siendo el cisplatino el principal quimioterápico inductor de ototoxicidad.

Palavras- clave: Cisplatino, Emisiones otoacústicas, Tratamiento farmacológico, Celulas ciliadas externas.

\section{INTRODUÇÃO}

A audição é fundamental à vida. Durante a infância há o desenvolvimento e aquisição da linguagem, que desempenha papel importante na comunicação e interações sociais (Gatto, 2007). Um dos principais distúrbios que podem interferir no desenvolvimento da linguagem é a deficiência auditiva, que acomete cerca de $60 \%$ da população mundial. No Brasil observa-se perda auditiva em $5,1 \%$ da população, segundo dados do Instituto Brasileiro de Geografia e Estatística (IBGE).

Kemp em 1978, foi quem observou inicialmente as emissões otoacústicas (EOA), definindo-as como uma liberação de energia sonora procedente da cóclea, a qual percorre a orelha média e chega ao meato acústico externo. Com isso, evidenciou-se que essas emissões se encontram presentes em todos os indivíduos que apresentam normalidade funcional da orelha. (Probst, 1990). Quando evocadas, as emissões são comumente classificadas em função do estímulo gerador: em transientes, estímulos frequências e por produtos de distorção. (Probst et al, 1991).

Devido as suas propriedades biomecânicas, as células externas têm capacidade de dois tipos de contração: rápida e lenta. Essa capacidade de contração constitui um amplificador coclear e tem grande importância no mecanismo de amplificação do estímulo sonoro para determinar o funcionamento das células ciliadas internas, que são as unidades receptoras e codificadoras cocleares. (Gattaz, 1997).

As EOA asseguram um diferencial no diagnóstico por se tratar de um teste prático que avalia a função coclear de forma segura e são sensíveis aos estágios iniciais de alterações e portanto, auxiliam no diagnóstico precoce. É importante saber que as emissões são registradas com limiar normal ou perda auditiva inferior a $30 \mathrm{~dB}$. (Coelho, 2010).

A técnica mais empregada e recomendada nas triagens auditivas tem sido as emissões otoacústicas evocadas transientes, por utilizar estímulos acústicos de fraca intensidade, abrangendo uma vasta gama de frequências (400 a $6400 \mathrm{~Hz})$. (Andrade, 2011).

Foi demonstrado através de estudos, que as EOA podem ser utilizadas como testes de triagem, com elevada sensibilidade em pacientes submetidos a terapia com as platinas. O tratamento quimioterápico contém a platina, representada por drogas como cisplatina e carboplatina, que pode ser utilizada para tratar diversos tumores como sarcoma osteogênico, blastoma medular, cânceres de testículo, útero e ovário, podendo apresentar efeitos adversos irreversíveis, como a otoxicidade, evoluindo com perdas auditivas 
permanentes em até 40 a 60\% das crianças que a utilizam (Parken, 2016). A toxicidade ocorre a partir da liberação de mediadores reativos de oxigênio, que causam dano celular e apoptose das células da orelha interna, incluindo células ciliadas do órgão de Corti. (Knight, 2017). A perda auditiva na sua maioria é do tipo neurossensorial, bilateral e progressiva, em altas frequências e associada a zumbido. Geralmente ocorrem cerca de algumas horas após a exposição a droga ou até uma semana após o tratamento, podendo ser parcial e temporária ou permanente (Broke, 2012).

A toxicidade varia de acordo com o tempo de exposição, a dose, o método e o número de ciclos programados. Alguns estudos associaram também pacientes com insuficiência renal, com nível elevados de creatinina e de melanina (negros e de olhos mais escuros), com maior risco de intoxicação (Bhavsar, 2017).

Um monitoramento adequado da audição se torna importante para a redução do impacto na função auditiva e uma tentativa de tratamento precoce nesses pacientes. Alguns países adotaram um sistema de monitoramento de função auditiva nos pacientes em uso de quimioterápicos, que inclui: história da doença, otoscopia, imitanciometria, audiometria tonal e otoemissões acústicas, sendo avaliadas até seis meses após o tratamento. (Caldas et al, 2015).

Dessa forma, o objetivo do presente estudo foi avaliar as emissões otoacústicas em pacientes submetidos a tratamentos quimioterápicos em hospital de referência em oncologia pediátrica do Pará.

\section{METODOLOGIA}

O estudo é do tipo longitudinal, prospectivo e de coorte. A pesquisa ocorreu no Hospital Oncológico Infantil Octávio Lobo e no Hospital Universitário Bettina Ferro de Souza (HUBFS), obtendo-se dados dos exames de emissões otoacústicas transientes, em pacientes atendidos no ambulatório de Oncologia Pediátrica do Hospital Oncológico Infantil Octávio Lobo.

A população estudada foi de pacientes atendidos no ambulatório de Oncologia Pediátrica do Hospital Oncológico Infantil Octávio Lobo, no período de outubro de 2018, com diagnóstico de neoplasia e submetido a tratamento quimioterápico. A amostra foi composta por 20pacientes que se encaixaram nos critérios de inclusão e forem eliminados pelos critérios de exclusão.

Foram usados critérios de inclusão: Pacientes atendidos no ambulatório de Oncologia Pediátrica no período outubro de 2018; com diagnóstico de neoplasia confirmada através de exames complementares; e pacientes que realizaram tratamento com drogas quimioterápicas no período de seis meses. Os critérios de exclusão foram ausência de diagnóstico de neoplasia mediante exames complementares e o diagnóstico de perda auditiva de qualquer grau ou tipo, previamente.

Os dados relativos a este trabalho foram coletados a partir da realização de exames de EOA transientes, dos pacientes atendidos no Hospital Oncológico Infantil Octávio Lobo, através do aparelho que capta otoemissões, modelo Otoread da marca Interacoustics, ao início do tratamento e durante o uso de quimioterápicos (média de 20 semanas de tratamento), através de formulários. Por se tratar de coleta de dados através de exames complementares, foi indispensável o Termo de Consentimento Livre e Esclarecido. A coleta dos dados somente se iniciou após avaliação pelo Comitê de Ética Médica em pesquisa com aprovação número 3.040.642. Foram coletados dados referentes à: sexo, idade, diagnóstico, identificação da droga antineoplásica e a dose utilizada, tempo do tratamento, otoscopia do paciente e exame de emissões otoacústicas. Os dados coletados foram lançados em uma planilha do Excel 2010, formando um banco de dados para posterior tratamento e análise estatística.

Todos os pacientes da presente pesquisa foram estudados segundo os preceitos da Declaração de Helsinque e do Código de Nuremberg, respeitadas as normas de pesquisa envolvendo seres humanos (Res. CNS 196/96) do Conselho Nacional de Saúde e após a aprovação do projeto de pesquisa pelo Comitê de Ética em Pesquisa da Universidade Federal do Pará. 
As informações da caracterização amostral foram apuradas em banco de dados elaborado no software Microsof ${ }^{\circledR}$ Office Exce $^{\circledR}$ 2010.Na aplicação da Estatística Descritiva, foram construídos tabelas e gráficos para apresentação dos resultados. A estatística analítica foi utilizada para avaliar os resultados das variáveis categóricas da amostra através dos Testes G e Qui-Quadrado. Aderência para tabelas univariadas e Testes G e Qui-Quadrado Independência para tabelas bivariadas. As estatísticas descritiva e analítica, foram realizadas no software BioEstat ${ }^{\circledR} 5.3$ (AYRES et al., 2010). Para a tomada de decisão, adotou-se o nível de significância $\alpha=0,05$ ou $5 \%$, sinalizando com asterisco $\left(^{*}\right)$ os valores significantes.

\section{RESULTADOS}

Foram avaliadas EOA transientes de vinte pacientes no início e durante o tratamento com quimioterápicos. Nos quais foram encontrados os seguintes resultados, de acordo com o protocolo de pesquisa.

Tabela 1 - Presença de emissões otoacústicas.

\begin{tabular}{ccc}
\hline EOA Transientes & Frequência & $\%$ \\
\hline \hline Presentes $^{*}$ & 12 & $60,0 \%$ \\
Ausentes & 7 & $35,0 \%$ \\
Inconclusivo & 1 & $5,0 \%$ \\
\hline Total & $\mathbf{2 0}$ & $\mathbf{1 0 0 , 0 \%}$ \\
\hline
\end{tabular}

Fonte: Protocolo de pesquisa, 2018

${ }^{*} \mathrm{p}=0.0106$ Teste $\mathrm{G}$ Aderência

Em relação a variável gênero, não foi encontrada incidência significativa na sua proporção, com predomínio do sexo masculino.

Foram avaliados dados referentes a presença ou ausência de EOA transientes, com prevalência significativa da presença das mesmas em $60 \%$ dos pacientes. Apenas um paciente mostrou resultado inconclusivo (Tabela 1). Foram coletados dados sobre os principais quimioterápicos utilizado no tratamento de diversas neoplasias, com prevalência de platinas e seus derivados em $50 \%$ dos paciente $(p<0.0001)$. (Tabela 2)

A relação da presença ou ausência de emissões otoacústicas de acordo com o quimioterápico utilizado foi analisada, observando a prevalência da presença de otoemissões em $60 \%$ dos pacientes em uso de platinas e seus derivados $(p<0.00001)$. (Tabela 3 ).

O tempo de tratamento e a exposição ao quimioterápico, também foram avaliados, podendo demonstrar, que o tempo não influencia diretamente na ausência de EOA (Tabela 4). 
Tabela 2 - Quimioterápicos em uso.

\begin{tabular}{ccc}
\hline Quimioterápico & Frequência & $\%$ \\
\hline \hline Platinas e Derivados* & 10 & $50,0 \%$ \\
Vincristina & 4 & $20,0 \%$ \\
Etoposide & 4 & $20,0 \%$ \\
Vinorelbine & 1 & $5,0 \%$ \\
Zometa & 1 & $5,0 \%$ \\
\hline Total & $\mathbf{2 0}$ & $\mathbf{1 0 0 , 0 \%}$ \\
\hline \hline
\end{tabular}

Fonte: Protocolo de pesquisa, 2018.

${ }^{*} \mathrm{p}<0.0001$ Teste G Aderência

Tabela 3 - Presença de EOA de acordo com quimioterápico.

\begin{tabular}{ccccccc}
\hline \multirow{2}{*}{ Quimioterápicos } & \multicolumn{5}{c}{ EOA Transientes } \\
\cline { 2 - 7 } & \multicolumn{2}{c}{ Presente } & \multicolumn{2}{c}{ Ausente } & \multicolumn{2}{c}{ Inconclusivo } \\
\hline \hline Platinas e Derivados & 6 & $60,0 \%$ & 4 & $40,0 \%$ & 0 & $0,0 \%$ \\
Vincristina & 2 & $50,0 \%$ & 2 & $50,0 \%$ & 0 & $0,0 \%$ \\
Etoposide & 4 & $100,0 \%$ & 0 & $0,0 \%$ & 0 & $0,0 \%$ \\
Vinorelbine & 0 & $0,0 \%$ & 1 & $100,0 \%$ & 0 & $0,0 \%$ \\
Zometa & 0 & $0,0 \%$ & 0 & $0,0 \%$ & 1 & $100,0 \%$ \\
\hline TOTAL & $\mathbf{1 2}$ & $\mathbf{6 0 , 0 \%}$ & $\mathbf{7}$ & $\mathbf{3 5 , 0}$ & $\mathbf{1}$ & $\mathbf{5 , 0} \%$ \\
\hline \hline
\end{tabular}

Fonte: Protocolo de pesquisa, 2018.

${ }^{*} p<0.0001$ Teste G Independência

Foram coletados também dados referentes a dosagem dos quimioterápicos utilizados, porém não foi possível aplicar teste estatístico, sem demonstrar no atual estudo, relação significativa da dosagem com a ausência ou presença de emissões otoacústicas. 
Tabela 4 - Tempo de exposição ao quimioterápico.

\begin{tabular}{ccccccc}
\hline \multirow{2}{*}{ Tempo de tratamento } & $(\mathbf{s e m})$ & \multicolumn{5}{c}{ EOA Transientes } \\
\cline { 2 - 7 } & & Presente & & Ausente & Inconclusivo \\
\hline \hline Início & 3 & $30,0 \%$ & 0 & $0,0 \%$ & 0 & $0,0 \%$ \\
10 sem & 2 & $20,0 \%$ & 0 & $0,0 \%$ & 0 & $0,0 \%$ \\
10 a 19 & 1 & $10,0 \%$ & 0 & $0,0 \%$ & 0 & $0,0 \%$ \\
20 a 29 & 1 & $10,0 \%$ & 3 & $33,3 \%$ & 0 & $0,0 \%$ \\
30 a 39 & 1 & $10,0 \%$ & 3 & $33,3 \%$ & 1 & $100,0 \%$ \\
40 a 49 & 2 & $20,0 \%$ & 2 & $22,2 \%$ & 0 & $0,0 \%$ \\
50 ou mais & 0 & $0,0 \%$ & 1 & $11,1 \%$ & 0 & $0,0 \%$ \\
\hline TOTAL & $\mathbf{1 0}$ & $\mathbf{5 0 , 0} \%$ & $\mathbf{9}$ & $\mathbf{4 5 , 0} \%$ & $\mathbf{1}$ & $\mathbf{5 , 0} \%$ \\
\hline \hline
\end{tabular}

Fonte: Protocolo de pesquisa, 2018

$p=0.1699$ Teste $G$ Independência

\section{DISCUSSÃO}

Foram avaliados vinte pacientes, observando-se predomínio do sexo masculino, com maior prevalência de EOA presentes. Dentre os quimioterápicos, prevaleceram as platinas e seus derivados. A relação entre a EOA com o quimioterápico utilizado, resultou em EOA presentes em pacientes em uso de platinas e derivados. Demonstrou- se que o tempo e a dose de medicação não influenciam diretamente o resultado.

Sakat e cols. (2018) observou que a perda de audição seria dose dependente do quimioterápico, pois quanto maior o tempo de exposição à droga e a dosagem, haveria maior possibilidade de degeneração e necrose das células ciliadas externas, o que não foi observado no presente estudo. Este achado divergente da literatura pode ser justificado pois $30 \%$ dos pacientes avaliados, ainda estavam no início do tratamento com cisplatina e apresentavam emissões transientes presentes.

No estudo de W van As (2016), houve uma comparação entre a dosagem de medicação utilizada, com resultado de maior perda auditiva naqueles pacientes expostos a dosagem superior a $400 \mathrm{mg} / \mathrm{m}^{2}$. Isso corrobora com nosso estudo, pois a dosagem máxima de cisplatina utilizada foi de $300 \mathrm{mg} / \mathrm{m}^{2}$, não apresentando valor significativo quando relacionada a presença ou ausência de emissões com a dose de medicação.

Rybak e cols. (2009) mostrou que a dosagem de medicação pode afetar o ouvido interno de forma diferente. Uma dose superior a $150 \mathrm{mg} / \mathrm{m} 2$ seria o suficiente para causar dando coclear, podendo inclusive haver perdas em sons de média frequência. Esse resultado demonstra que uma quantidade inferior da droga pode levar a consequências, discordando do achado em nosso estudo.

No estudo de Kuo e cols. (2007), foi possível observar que muitos pacientes apresentaram perda de audição cerca de dois anos após o tratamento, observando que o efeito da dosagem e da droga pode ser cumulativo. As emissões otoacústicas podem ser usadas como avaliação objetiva da função das células ciliadas externas, com elevada sensibilidade para avaliar perda de audição precocemente. O estudo de Knight 
(2016) demonstrou uma elevada sensibilidade no uso de emissões otoacústicas, superior até mesmo aos testes auditivos convencionais no início das perdas auditivas. Rayback (2009), observou que $90,5 \%$ dos pacientes apresentavam perdas nas frequências superiores a $8000 \mathrm{~Hz}$ e que o estudo de emissões otoacústicas transientes apresentam uma elevada sensibilidade para essa captação, demostrando-se novamente que podem ser utilizadas como bom método de rastreio e acompanhamento de pacientes em tratamento.

Brock e cols. (2012) atentou para possível predisposição genética em alguns pacientes em uso de platinas que evoluíram com perda de audição. Dois genes são apontados como os principais predisponentes, são eles o gene tiopurina S-metiltransferase (TPMT) e catecol O-metiltransferase (COMT), que estão relacionados a perdas auditivas severas e uso da cisplatina. Esse achado justifica a presença de emissões otoacústicas em alguns pacientes em uso da cisplatina, que podem não apresentar predisposição genética para a perda auditiva.

O estudo de Van den Berg (2016), observou uma elevada variação na prevalência de perda de audição após uso de platinas, indo de 1 a $90 \%$. Isso nos ajuda mais uma vez a definir que não são todos os pacientes expostos ao tratamento, que apresentarão a complicação.

Chirtes e Albu (2014) relataram que o tempo de exposição ao quimioterápico não apresenta relação com a perda de audição, onde uma simples exposição pode ser significativa. Nosso estudo não encontrou significado estatístico no tempo de exposição a droga.

Apesar de conclusões importantes verificadas no trabalho, apresentamos um número pequeno de pacientes na amostra, diferente dos demais trabalhos avaliados, que foram feitos em um período de anos, com uma amostra de pacientes consideravelmente maior. Mesmo com tal fato, pode-se concluir resultados significativos.

\section{CONCLUSÃO}

O presente estudo apontou que as otoemissões estavam presentes na maioria dos pacientes que estavam em início do tratamento, e ausentes naqueles em estádios avançados de tratamento. Não encontramos relação direita com o tempo de exposição e a dosagem da droga utilizada, sendo a cisplatina e seus derivados o principal quimioterápico indutor de ototoxicidade. É importante destacar que a perda de audição com uso de quimioterápicos acomete com maior incidência crianças, podendo causar perda auditiva pré lingual com impacto importante na vida acadêmica e social desses pacientes. Sendo assim, torna-se importante rastrear e acompanhar a audição dos pacientes em tratamento quimioterápico afim de prevenir um maior dano auditivo.

\section{REFERÊNCIAS}

1. ANDRADE IFC, et al. Transient evoked otoacoustic emissions (teoe): newborn hearing screening program protocols. RevistaCefac São Paulo, 2011.

2. BASSETTO MCA, et al. Transient Evoked otoacustic emissions (TEOAE): response amplitude in term and preterm neonates. Revista Brasileira de Otorrinolaringologia 69; Parte 1 Janeiro/ Fevereiro 2003.

3. BHAVSAR AP, et al. Pharmacogenetic variants in TPMT alter cellular responses to cisplatin in inner ear cell lines. April 13, 2017 https://doi.org/10.1371/journal.pone.0175711.

4. BROCK PR, et al. Platinum-Induced Ototoxicity in Children: A Consensus Review on Mechanisms, Predisposition, and Protection, Including a New International Society of Pediatric Oncology Boston Ototoxicity Scale. Journal of Clinical Oncology. 2012;30(19):2408-2417. doi:10.1200/JCO.2011.39.1110.

5. CHRISTES F, ALBU S. Prevention and Restoration of Hearing Loss Associated with the Use of Cisplatin. BioMed Research International. 2014;2014:925485. doi:10.1155/2014/925485.

6. COELHO MSB, et al. Otoacoustic Emissions in the differential diagnosis of the noise-induced auditory losses. Revista CEFAC. 2010 Nov-Dez; 12(6):1050-1058. 
7. CRABB SJ, et al. COAST (Cisplatin ototoxicity attenuated by aspirin trial): A phase II double-blind, randomised controlled trial to establish if aspirin reduces cisplatin induced hearing-loss. European Journal of Cancer. 2017;87:75-83. doi:10.1016/j.ejca.2017.09.033.

8. $\mathrm{KIM} \mathrm{KH}$, et al. Evaluating protective and therapeutic effects of alpha-lipoic acid on cisplatin-induced ototoxicity. Cell Death \&Disease. 2018;9(8):827. doi:10.1038/s41419-018-0888-z.

9. KNIGHT KR, et al. Group-Wide, Prospective Study of Ototoxicity Assessment in Children Receiving Cisplatin Chemotherapy (ACCL05C1): A Report From the Children's Oncology Group; DOI: 10.1200/JCO.2016.69.2319 Journal of Clinical Oncology 35, no. 4 (February 2017) 440-445.

10. LIBERMAN PHP et al. Audiological profile of patients treated for childhood cancer. Brazilian Journal of Otorhinolaryngology, 2016-11-01, Volume 82, Edição 6, Páginas 623-629, Copyright (C) 2016. Associação Brasileira de Otorrinolaringologia e Cirurgia Cérvico-Facial.

11. OLIVEIRA PF, et al. Cancer treatment in determination of hearing loss. 1808-8694/C 2015 Associação Brasileira de Otorrinolaringologia e Cirurgia Cérvico-Facial. Published by Elsevier Editora Ltda. All rights reserved.

12. PAKEN J, et al. Cisplatin-Associated Ototoxicity: A Review for the Health Professional. Journal of Toxicology Volume 2016 (2016), Article ID 1809394, 13 p.

13. PIAZZI PR, GATTAZ G. Emissões otoacústicas: conceitos básicos e aplicações clínicas. International Archivies of otorhinolaryngology. V 1, num. 2, Abr/Jun, 1997.

14. RAYBAK LP, et al. Cisplatin Ototoxicity and Protection: Clinical and Experimental Studies. The Tohoku journal of experimental medicine. 2009;219(3):177-186.

15. SAKAT MS, et al. The effectiveness of eugenol against cisplatin-induced ototoxicity. Braz J Otorhinolaryngol. 2018. https://doi.org/10.1016/j.bjorl.2018.07.007.

16. TOCHETTO TM. Infantile hearing loss: implications and solutions. Revista Cefac São Paulo, v. 9, n. 1, 110-15, jan-mar, 2007.

17. VAN As JW, et al. Platinum-induced hearing loss after treatment for childhood cancer. Cochrane Database of Systematic Reviews 2016, Issue 8. Art. No.: CD010181. DOI: 10.1002/14651858.CD010181.pub2. 